\title{
Self-organized propagation patterns from dynamic self-assembly in monolayers
}

\author{
Josep Claret, Jordi Ignés-Mullol, Ramon Reigada, and Francesc Sagués* \\ Departament de Química Física, Universitat de Barcelona, Martí i Franquès 1, E-08028 Barcelona, Spain \\ Joaquim Crusats \\ Departament de Química Orgànica, Universitat de Barcelona, Martí i Franquès 1, E-08028 Barcelona, Spain
}

(Received 20 April 2005; published 27 February 2006)

\begin{abstract}
Propagation of localized orientational waves, as imaged by Brewster angle microscopy, is induced by low intensity linearly polarized light inside axisymmetric smectic- $C$ confined domains in a photosensitive molecular thin film at the air/water interface (Langmuir monolayer). Results from numerical simulations of a model that couples photoreorientational effects and long-range elastic forces are presented. Differences are stressed between our scenario and the paradigmatic wave phenomena in excitable chemical media.
\end{abstract}

DOI: 10.1103/PhysRevE.73.026225

PACS number(s): 47.54.- r, 68.18.-g, 81.16.Dn

Maintained away from equilibrium, many natural and laboratory tailored systems provide striking examples of dynamic self-organizing structures. In particular, experimental and theoretical investigations of nonequilibrium patterns in physical and chemical contexts have often focused on reaction-diffusion mechanisms [1,2]. Moreover, this kind of description has been sometimes invoked as a possible underlying concept for some aspects of biological selforganization and morphogenesis [3]. Biology, however, is the realm of spatially confined complex fluids, whose behavior is largely different from ideally low-density fluid phases or even compared to freely condensed soft materials [4]. In many instances, biological fluids feature distinctive characteristics of self-assembly. Although a rather fuzzy concept [5], we would define self-assembly as the spontaneous capacity of properly designed molecular components to reversibly arrange through their mutual interactions into hierarchical supramolecular structures. Thus a natural issue to address is whether some of the most prototypic reaction-diffusion modes of self-organization could similarly arise from the dynamic self-assembling of labile and low-dimensional condensed systems [6].

Langmuir monolayers (monomolecular films of amphiphilic, water-insoluble molecules at the air/water interface) are model systems with which to study ordering and phase transitions in two dimensions [7]. Choosing appropriately an amphiphile with strong interactions between hydrophobic tails, typically a substance that is also a bulk mesogen, it should be possible to find labile mesophases suitable to study dynamical patterns in two dimensions as a response to modest external perturbations.

In this paper, we report experimental observations of well-resolved localized propagation patterns in carefully tailored photoresponsive monolayers. Moreover, a model based on the interplay between the orientational order parameter and a scalar concentration field reveals that these are indeed self-organized patterns intimately tied to light-mediated dynamical changes in the self-assembly of these monolayer systems.

\footnotetext{
*Electronic address: f.sagues@ub.edu
}

The chosen amphiphile is a photoisomerizable cis/trans azobenzene derivative, [4-(4-[(4-octylphenyl)azo]phenoxy)butanoic acid], $8 \mathrm{Az} 3 \mathrm{COOH}$ hereforth. The crucial aspect of the monolayer preparation consists in spreading a cis/trans photostationary mixture of the isomers [8-10]. Given their different physico-chemical properties, they spontaneously phase separate, forming circular droplets of mesogenic trans molecules surrounded by an isotropic matrix of cis molecules. The structure and dynamics of this system is studied in real time with Brewster angle microscopy (BAM). This technique reveals that, prior to irradiation, stretched molecules inside the domains have a nearly constant tilt (with respect to the direction normal to the monolayer) and compose an axisymmetric azimuthal distribution organized around a central point defect. Within these domains, localized orientational waves are going to form, propagate, and fade away, after continued irradiation with polarized light of appropriate intensity and wavelength.

Previous work by Tabe et al. [11,12], referring, however, to pure trans monolayers, reported changes in the molecular orientational order under polarized illumination and the nucleation of traveling photoaligned localized domains under sustained forcing. Nevertheless, their study focused on stripelike patterns of modulated tilt and azimuth or in configurations with poorly defined initial conditions not amenable to direct BAM resolution, thus rendering their results rather inconclusive. Phenomenological models have been proposed recently [13-16], allegedly relating the observed behavior to the photoisomerizable trans/cis properties of these compounds.

Our experiments were conducted in a Teflon through at $35^{\circ} \mathrm{C}$ and a lateral pressure of $2 \mathrm{mN} \mathrm{m}^{-1}$. BAM images were recorded with the analyzer set at $60^{\circ}$ counterclockwise with respect to the plane of incidence, which contains the $y$ axis of the images. After spreading, trans droplets are formed within a few minutes. Monolayers were then irradiated with linearly polarized light (horizontal direction in the pictures shown below) [17]. Within seconds after illumination, a photoalignment of domains took place dividing them into two semicircles with molecular tails pointing inwards perpendicularly to the polarization direction. Both halves are sepa- 

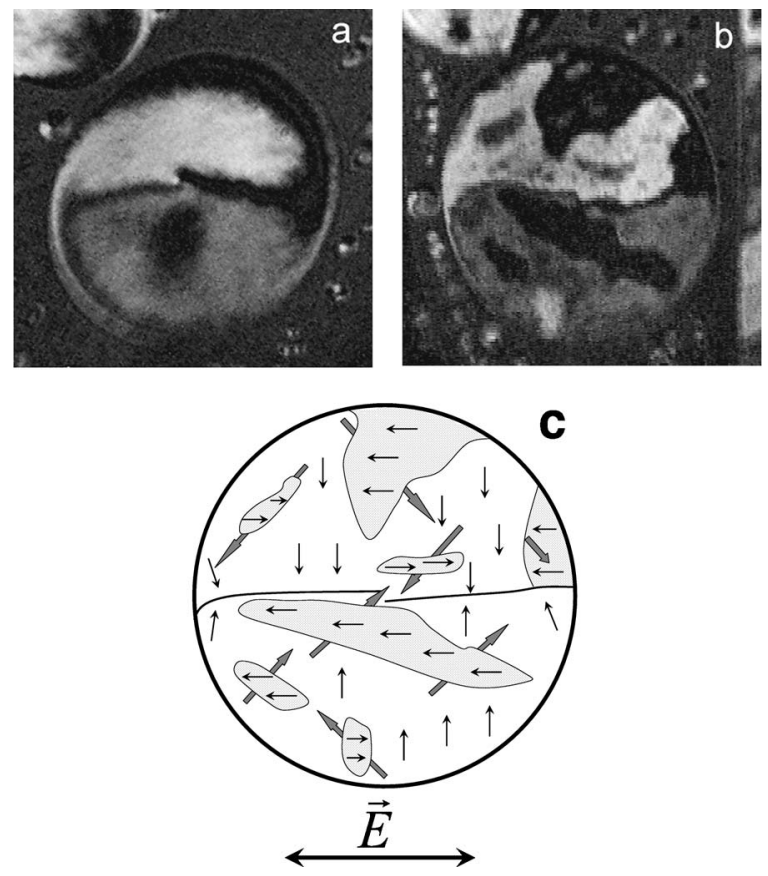

FIG. 1. (a) BAM image of a photoaligned domain after $37 \mathrm{~s}$ of irradiation of a droplet with polarized light. (b) BAM image showing traveling waves propagating inside the photoaligned domain [20]. The time elapsed between both pictures is $156 \mathrm{~s}$. The width of the pictures is $600 \mu \mathrm{m}$. The illumination power is $0.8 \mathrm{~mW} \mathrm{~cm}^{-2}$. (c) Scheme representing the azimuthal orientation of trans- $8 \mathrm{Az} 3 \mathrm{COOH}$ molecules inside the waves (thin arrows) and their advancing directions (wide arrows) of the waves of (b).

rated by a dividing line aligned roughly along the polarization direction $[18,19]$ [Fig. 1(a)].

The final stages of such photoalignment process often lead to the appearance of the propagating spots in which we are interested here. They nucleated as localized distortions of the mostly homogeneous (and normal to polarization) azimuthal field, and moved towards the dividing line [Fig. 1(b)]. The origin of these traveling spots is rather uncertain. As a matter of fact, monolayers, under our conditions of observation, exhibited a pronounced flickering in their reflectivity, a signature of their orientational lability under thermal noise $[10,21]$. Such internal fluctuations appeared sometimes to be strong enough to randomly trigger waves. Other observations were indicative of their origin from small heterogeneities in the orientational field, principally near the boundaries of the droplet. The question of an externally controlled nucleation of the spots remains an open issue to be investigated in the future. Simultaneous and repeated nucleation from different locations was commonly observed, although the limited space available for propagation within the segmented droplet normally impeded the organization of regular trains. In fact this difficulty was somewhat overcome when illuminating droplets with their point defect at the boundary leading to a quasiuniform photoalignment within the entire droplet (Fig. 2).

Some remarkable features are unambiguously established from our resolved BAM observations. (i) Waves propagate as localized azimuthal distortions with molecular tails nearly
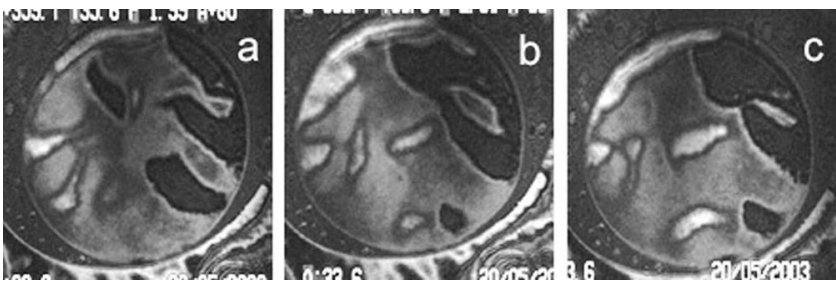

FIG. 2. Successive BAM images of traveling waves in a photoaligned domain with the defect at the boundary (upper part) [20]. The time elapsed between pictures (a) and (b) is $6 \mathrm{~s}$, and between (b) and (c) is $5 \mathrm{~s}$. The width of the pictures is $930 \mu \mathrm{m}$. The illumination power is $0.8 \mathrm{~mW} \mathrm{~cm}^{-2}$.

parallel to the polarization direction [Fig. 1(c)], traveling on an underlying texture of perpendicular orientations. Below we will demonstrate, indeed, that the nature of the waves is not purely orientational, but involves a compositionlike modulation as well. (ii) The traveling direction is always fixed inward at nearly $\pi / 4$ with respect to the polarization direction. (iii) When a traveling wave meets the dividing line they both merge if the azimuth of the wave matches that at the center of the wall at the meeting point. Otherwise, they do not merge and the wave fades away [see Fig. 3(c)]. (iv) When light is switched off, waves immediately stop and their reflectivity gradually relaxes to that of the underlying texture. This means that waves fade away at place before the whole droplet recovers the stable configuration in absence of light. (v) The last important feature we want to emphasize is the behavior of waves with oppositely oriented molecules when they approach. Contrary to other wave propagation scenarios (see discussion below), a process of mutual annihilation does not occur (Fig. 3). Depending on the size of the smaller approaching spot, it either slows down to give way to the larger one or it simply fades away if sufficiently small.

To interpret the observed wave phenomena we propose a theoretical model built from the interplay of photoinduced and elastic effects $[13,14]$. Since trans/cis compositional changes in the monolayer during the course of the experiments are negligible [19], we consider that the droplets remain composed mainly of trans-molecules. Under linearly polarized illumination, two concurrent processes permit such stretched molecules to reduce their excitability: a photomigration route which drives the azobenzene molecular axis
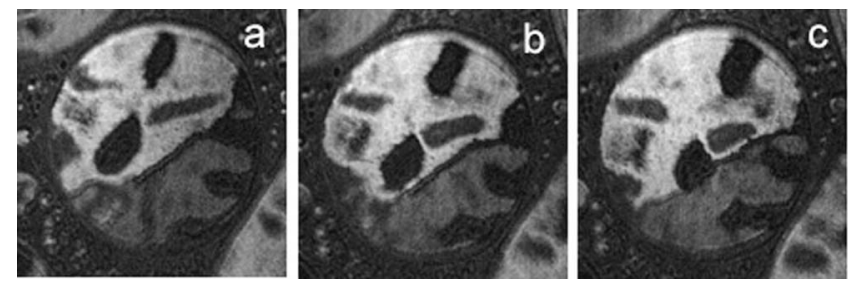

FIG. 3. Successive BAM images showing the absence of annihilation between traveling waves [20]. The central defect on the dividing line is shifted to the left. As a result, the left (darker) wave merges with the dividing line while the right one does not. The time elapsed between pictures (a) and (b) is $2 \mathrm{~s}$, and between (b) and (c) is $5 \mathrm{~s}$. The width of the pictures is $675 \mu \mathrm{m}$. The illumination power is $1 \mathrm{~mW} \mathrm{~cm}^{-2}$. 
perpendicular to the polarization direction $[19,22,23]$, and, simultaneously, a mechanism of light-induced molecular aggregation, which shifts the absorption band of the monomers thus reducing their photomigration ability $[10,19,24,25]$. On the other hand, the texture of the droplets is accounted for by a free-energy functional incorporating elastic effects. In its simplest form the whole description is formulated with two spatio-temporal dependent state variables: $\varphi(x, y ; t)$ stands for the azimuth variable (measured with respect to the polarization direction), and $c(x, y ; t)$ denotes the molecular fraction of nonaggregated trans-molecules. Kinetic and relaxational equations are readily written down as a coupled scheme $[18,19]$,

$$
\begin{gathered}
\frac{\partial c}{\partial t}=-\left(k_{1} \cos ^{2} \varphi+k_{2}\right) c+k_{2}, \\
\frac{\partial \varphi}{\partial t}=-\Gamma \frac{\delta \mathcal{F}[\varphi, c]}{\delta \varphi}+\zeta,
\end{gathered}
$$

where $k_{1} \cos ^{2} \varphi$ and $k_{2}$ stand for the (anisotropic and light intensity dependent) aggregation and (thermal) disaggregation rate constants, $\Gamma$ involves the typical time scale for molecular reorientations, and $\zeta$ introduces thermal fluctuations through a noise term satisfying the usual fluctuationdissipation relation $\left\langle\zeta_{i}(t) \zeta_{j}\left(t^{\prime}\right)\right\rangle=2 \Gamma k_{B} T \delta_{i j} \delta\left(t-t^{\prime}\right)$. The freeenergy functional for a single droplet, incorporating bulk and boundary contributions, reads $[18,19]$

$$
\begin{aligned}
\mathcal{F}= & \int_{\text {bulk }}\left[\frac{K_{s}}{2}\left(\vec{\nabla}_{x y} \cdot \vec{a}\right)^{2}+\frac{K_{b}}{2}\left|\vec{\nabla}_{x y} \times \vec{a}\right|^{2}\right. \\
& \left.+\lambda(1-c)\left(\vec{\nabla}_{x y} \cdot \vec{a}\right)\right] d x d y-\int_{\text {boun }} \beta\left(\vec{a} \cdot \overrightarrow{n_{b}}\right) d l,
\end{aligned}
$$

where $\vec{a}=(\cos \varphi, \sin \varphi)$ is the molecular in-plane unit director, $K_{s}$ and $K_{b}$ correspond to the splay and bend elastic constants, $\lambda$ is the coefficient of the coupled concentration-splay term, $\overrightarrow{n_{b}}$ is the unit boundary normal vector, and $\beta$ corresponds to the phenomenological parameter accounting for the interaction between inner and outer media. Notice that the crucial term incorporating the coupling between the $\varphi$ and $c$ fields is the linear divergence term, allowed by the symmetries of the monolayer and whose effect is rendered nontrivial by the nonuniform composition field [26].

In Fig. 4 we present some direct numerical simulations of the dynamical scheme proposed above, by discretizing Eqs. (1) and (2) in a circular domain, and numerically solving them starting from an already photoaligned structure. We note the good agreement between the simulation and the experimental results in what concerns the main trends of this
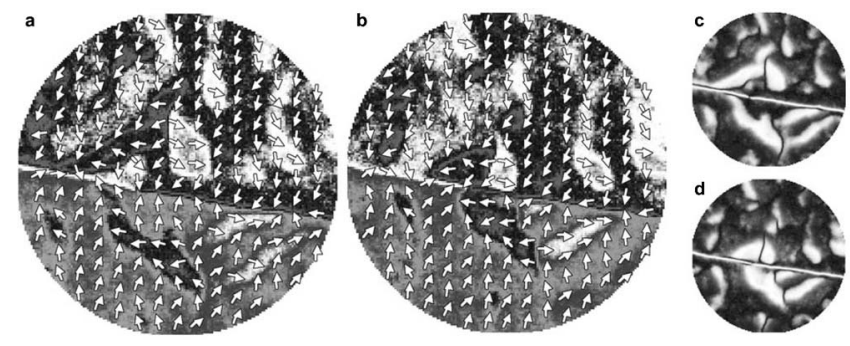

FIG. 4. Numerical simulations of model Eqs. (1)-(3). Two successive snapshots for a photoaligned droplet showing the propagation of orientational [(a) and (b)] and compositional [(c) and (d)] pulses. In (a) and (b) arrows represent the in-plane molecular orientation and the gray color emulates the BAM observations [20]. In (c) and (d), brighter regions stand for larger fractions of aggregated species $(c<1)$. The set of parameters is $K_{b}=0.8, k_{B} T=2.5$ $\times 10^{-3}[21], k_{1}=50, k_{2}=200, \lambda=600$, and $\beta=8$ in the unit system where $K_{s}=R$ (droplet radius) $=\Gamma=1$.

self-organized phenomenon, namely, both the azimuthal field inside the traveling waves and the advancing direction of the latter. In addition, numerical simulations reveal that the orientational wave propagates slightly ahead of the composition modulation.

A final comment is worth making considering the analogies and differences between our reported results and other scenarios of wave propagation phenomena. When compared to reaction-diffusion schemes typical of active chemical media, both the principles and the main characteristics of wave propagation are indeed different. Here, waves are anisotropically propagating structures triggered by orientational inhomogeneities and do not involve the minimum pair of disparate time scales for evolving excitatory and recovery variables typical of free or forced excitable media [27]. Rather, the "soft matter" waves reported here result from the dynamic and light-modified self-assembling properties of the monolayer. A clear signature of this distinctive character can be found, for instance, in the peculiar behavior, lacking annihilation of interacting waves in our system. Moreover, solitons in bulk liquid crystals, known to exist under rotating magnetic fields in the form of the so-called Bloch walls $[28,29]$, are also different at least in two respects: they annihilate upon contact and, more fundamentally, since involving $3 d$ materials they result from more energetically demanding torque, Freedericksz-like mechanisms as compared to the "soft" photoexcitation of the monolayers considered here.

This work was supported by SEID through Project No. BQU2003-05042-C02-01 and by DURSI through Project No. 2001SGR00045. J.I.-M., R.R., and J.Cr. acknowledge support from Ramón y Cajal Program (MCyT). 
[1] M. C. Cross and P. C. Hohenberg, Rev. Mod. Phys. 65, 851 (1993).

[2] A. De Wit, Adv. Chem. Phys. 109, 435 (1999).

[3] A. J. Koch and H. Meinhardt, Rev. Mod. Phys. 66, 1481 (1994).

[4] As an example we mention the question of microtubule formation in J. Tabony, Science 264, 245 (1994); J. Tabony, N. Glade, J. Demongeot, and C. Papaseit, Langmuir 18, 7196 (2002).

[5] G. M. Whitesides and B. Grzybowski, Science 295, 2418 (2002).

[6] A. Mikhailov and G. Ertl, Science 272, 1596 (1996).

[7] V. M. Kaganer, H. Möhwald, and P. Dutta, Rev. Mod. Phys. 71, 779 (1999).

[8] J. Crusats, R. Albalat, J. Claret, J. Ignés-Mullol, and F. Sagués, Langmuir 20, 8668 (2004).

[9] R. Reigada, E. Abad, J. Crusats, J. Claret, J. Ignés-Mullol, and F. Sagués, J. Chem. Phys. 121, 9066 (2004a).

[10] J. Ignés-Mullol, J. Claret, R. Albalat, J. Crusats, R. Reigada, M. T. Martín-Romero, and F. Sagués, Langmuir 21, 2948 (2005).

[11] Y. Tabe and H. Yokoyama, Langmuir 11, 4609 (1995).

[12] Y. Tabe, T. Yamamoto, and H. Yokoyama, New J. Phys. 5, 132 (2003).

[13] R. Reigada, F. Sagués, and A. S. Mikhailov, Phys. Rev. Lett. 89, 038301 (2002).

[14] R. Reigada, A. S. Mikhailov, and F. Sagués, Phys. Rev. E 69, 041103 (2004).

[15] T. Okuzono, Y. Tabe, and H. Yokoyama, Phys. Rev. E 69, 050701(R) (2004).

[16] T. Okuzono, Y. Tabe, and H. Yokoyama, Colloids Surf., B 38,
115 (2004).

[17] The light source is a $50 \mathrm{~W}$ halogen lamp, filtered through a band pass filter ( $480 \mathrm{~nm} \pm 5 \mathrm{~nm}$, Lambda) with power density in the range $0.5-5 \mathrm{~mW} \mathrm{~cm}^{-2}$.

[18] F. Sagués, R. Albalat, R. Reigada, J. Crusats, J. Ignés-Mullol, and J. Claret, J. Am. Chem. Soc. 127, 5296 (2005).

[19] J. Crusats, R. Albalat, J. Claret, J. Ignés-Mullol, R. Reigada, and F. Sagués, J. Chem. Phys. 122, 44722 (2005).

[20] See EPAPS Document No. E-PLEEE8-73-177602 for movie files corresponding to the experiments reported in this article. For more information on EPAPS, see http://www.aip.org/ pubservs/epaps.html

[21] A. Feder, Y. Tabe, and E. Mazur, Phys. Rev. Lett. 79, 1682 (1997).

[22] M. Schönhoff, M. Mertesdorf, and M. Lösche, J. Phys. Chem. 100, 7558 (1996).

[23] M. Kreuzer, E. Benkler, D. Paparo, G. Casillo, and L. Marrucci, Phys. Rev. E 68, 011701 (2003).

[24] M. K. Durbin, A. Malik, A. G. Richter, C. J. Yu, R. Eisenhower, and P. Dutta, Langmuir 14, 899 (1998).

[25] J. M. Pedrosa, M. T. Martín-Romero, L. Camacho, and D. Möbius, J. Phys. Chem. 106, 2583 (2002).

[26] Notice that if the prefactor of the divergence term would be uniform, the whole term could be integrated to a pure boundary contribution.

[27] R. Kapral and K. Showalter, Chemical Waves and Patterns (Kluwer Academic Publishers, Dordrecht, 1995).

[28] K. B. Migler and R. B. Meyer, Phys. Rev. Lett. 66, 1485 (1991).

[29] K. B. Migler and R. B. Meyer, Physica D 71, 412 (1994). 